\title{
A sharp estimate of the measure of the set of divergence of multiple orthogonal Fourier series
}

\author{
by \\ M. I. Dyachenko (Madrid and Moscow) and \\ K. S. KaZARIAN (Madrid and Yerevan)
}

\begin{abstract}
The aim of this paper is to obtain sharp estimates from below of the measure of the set of divergence of the $m$-fold Fourier series with respect to uniformly bounded orthonormal systems for the so-called $G$-convergence and $\lambda$-restricted convergence. We continue the study begun in a previous work.
\end{abstract}

1. Introduction. The purpose of the present paper is to generalize to the multi-dimensional case the following two theorems proved by one of the authors [3].

Theorem A. Let $\Phi=\left\{\varphi_{n}\right\}_{n=1}^{\infty}$ be an orthonormal system on $[0,1]$ uniformly bounded by $M \geq 1$. Then there exists a function $f \in L([0,1])$ whose Fourier series diverges at every point of some measurable set $E$ with $\mu(E) \geq 1 / M^{2}$.

Theorem B. Let $M>1$ and let $F \subset[0,1]$ be measurable with $\mu(F)=$ $1-1 / M^{2}$. Then there exists a complete orthonormal system $\left\{\eta_{n}\right\}_{n=1}^{\infty}$ on $[0,1]$, uniformly bounded by $M$, such that the Fourier series of any integrable function $f \in L([0,1])$ with respect to this system converges to $f$ almost everywhere on $F$.

A detailed survey of the results about divergent Fourier series for the trigonometric and other orthonormal systems (ONS) can be found in [6] and [3]. We would like to mention that the starting point for the study of divergent orthogonal Fourier series was Kolmogorov's [5] proof of the existence of an almost everywhere divergent Fourier series with respect to the trigonometric system.

2000 Mathematics Subject Classification: Primary 42B08; Secondary 40B05.

Research of the first author was partially supported by grant SAB2000-0002 of the Secretaria de Estado de Educación y Universidades del Ministerio de Educación, Cultura y Deporte de España and by grant RFFI 02-01-00315.

Research of the second author was supported by grant BFM2001-0189. 
Before presenting our results we make a few remarks on the above theorems. The reader should observe that in the one-dimensional case the exact estimate from below of the measure of the set of divergence for a Fourier series is the same in the classes of uniformly bounded complete orthonormal systems (CONS) and uniformly bounded ONS. For $m$-fold series, as will be shown in the present paper, the corresponding estimates are different. However, we show that in both cases the estimates are sharp.

Another new phenomenon in the multi-dimensional case concerns sets of positive measure where some complete uniformly bounded orthonormal system can converge almost everywhere. According to Theorem B for any measurable set with incomplete measure there exists a uniformly bounded CONS such that the given set has the desired property. In the multi-dimensional case, it will be shown that not every set of positive measure can be a set of convergence for $m$-fold Fourier series with respect to a uniformly bounded CONS.

It is well known that in the multi-dimensional case one can consider various partial sums of the Fourier series of an integrable function. The aim of this paper is to obtain sharp estimates from below for the measure of the set of divergence of the $m$-fold Fourier series with respect to uniformly bounded orthonormal systems for the so-called $G$-convergence and $\lambda$-restricted convergence, which will be defined in the next section. $G$-convergence and $\lambda$ restricted convergence are more general than, respectively, the spherical and cubical convergence for $m$-fold series. In a previous work [1] the authors have studied the same question for cubic partial sums. In the next section, after giving the precise notation, we comment on the results obtained in that work.

2. Notation and results. Let $\Phi=\left\{\varphi_{n}(x)\right\}_{n=1}^{\infty}$ be an orthonormal system (ONS) defined on $[0,1]$. Then for $m \geq 2$, we denote by $\Phi(m)=$ $\left\{\varphi_{\mathbf{n}}(\mathbf{x})\right\}_{\mathbf{n} \in \mathbb{N}^{m}}$, where $\varphi_{\mathbf{n}}(\mathbf{x})=\varphi_{n_{1}}\left(x_{1}\right) \cdot \ldots \cdot \varphi_{n_{m}}\left(x_{m}\right)$, the so-called product system of $\Phi$. If we suppose that the system $\Phi$ is complete then it is evident that $\Phi(m)$ is a complete orthonormal system on $[0,1]^{m}$.

For every $f \in L\left([0,1]^{m}\right)$, one can consider its Fourier expansion with respect to the system $\Phi(m)$, i.e. the $m$-fold Fourier series

$$
\sum_{\mathbf{n}=\mathbf{1}}^{\infty} a_{\mathbf{n}}(f) \varphi_{\mathbf{n}}(\mathbf{x}) \equiv \sum_{n_{1}=1}^{\infty} \ldots \sum_{n_{m}=1}^{\infty} a_{\mathbf{n}}(f) \varphi_{\mathbf{n}}(\mathbf{x}),
$$

where

$$
a_{\mathbf{n}}(f)=\int_{[0,1]^{m}} f(\mathbf{t}) \varphi_{\mathbf{n}}(\mathbf{t}) d \mathbf{t} \quad \text { for } \mathbf{n}=\left(n_{1}, \ldots, n_{m}\right) \in \mathbb{N}^{m}
$$

In the multi-dimensional case, for different types of partial sums of the series (1) the results can be quite different. In many questions of convergence 
of multiple series, if one deals with the so-called cubic partial sums the results are very close to the one-dimensional case. For the series (1) the cubic partial sums are defined as follows:

$$
S_{N}^{\Phi}(f)\left(x_{1}, \ldots, x_{m}\right)=\sum_{n_{1}=1}^{N} \ldots \sum_{n_{m}=1}^{N} a_{\mathbf{n}}(f) \varphi_{\mathbf{n}}(\mathbf{x}),
$$

where $N \in \mathbb{N}$. If the partial sums (2) converge in some sense (pointwise on a given set, in measure, in some metric) as $N \rightarrow \infty$ then the series (1) is said to converge cubically in the same sense. Otherwise, it diverges cubically in the relevant sense.

We are going to study multi-variate series with respect to an ONS $\Phi=$ $\left\{\varphi_{n}\right\}_{n=1}^{\infty}$ defined on $[0,1]$ which satisfies

$$
\left|\varphi_{n}(x)\right| \leq M \quad \text { almost everywhere on }[0,1] \text { for all } n \geq 1 \text {. }
$$

In what follows, we will denote by $\chi_{E}(\cdot)$ the characteristic function of the set $E$. The Lebesgue measures on $\mathbb{R}$ and $\mathbb{R}^{m}, m \geq 2$, will be denoted by $\mu$ and $\mu_{m}$, respectively.

In a previous article [1] we have obtained the following results:

TheOREM C. Let $\Phi=\left\{\varphi_{n}(x)\right\}_{n=1}^{\infty}$ be a complete orthonormal system on $[0,1]$ which satisfies $(3)$, let $m \geq 2$ and let $\Phi(m)$ be the corresponding product system. Then there exists a function $f(\mathbf{x}) \in L\left([0,1]^{m}\right)$ whose Fourier series with respect to $\Phi(m)$ diverges cubically everywhere on some measurable set $\mathcal{H} \subset[0,1]^{m}$ with $\mu_{m}(\mathcal{H}) \geq 1-\left(1-1 / M^{2}\right)^{m}$.

Theorem D. Let $\Phi=\left\{\varphi_{n}(x)\right\}_{n=1}^{\infty}$ be an orthonormal system on $[0,1]$ which satisfies (3), let $m \geq 2$ and let $\Phi(m)$ be the corresponding product system. Then there exists a function $f(\mathbf{x}) \in L\left([0,1]^{m}\right)$ whose Fourier series with respect to $\Phi(m)$ diverges cubically everywhere on some measurable set $\mathcal{H}_{0} \subset[0,1]^{m}$ with $\mu_{m}\left(\mathcal{H}_{0}\right) \geq 1 / M^{2 m}$.

The following example shows that the estimate of the measure of the set of divergence in Theorem $\mathrm{D}$ cannot be strengthened. For any $M>1$ one can define the following orthonormal system $\Psi$. Let

$$
\psi_{n}(t)= \begin{cases}M \cdot W_{n}\left(M^{2} t\right) & \text { if } t \in\left[0, M^{-2}\right) \\ 0 & \text { if } t \in\left[M^{-2}, 1\right]\end{cases}
$$

where $\left\{W_{n}\right\}_{n=1}^{\infty}$ is the Walsh system. Evidently the Fourier series with respect to the system $\Psi(m)$ converges on $[0,1]^{m} \backslash\left[0, M^{-2}\right)^{m}$ for any regular method of summation, in particular in any sense considered in this paper, and $\mu_{m}\left([0,1]^{m} \backslash\left[0, M^{-2}\right)^{m}\right)=1-1 / M^{2 m}$.

The sharpness of the estimate of the measure of the set of divergence in Theorem $\mathrm{C}$ is more complicated. Luckily the example constructed in [3] for 
this purpose in the one-dimensional case works also in the multi-dimensional case. In [1] we proved the following

TheOREM E. For any $M>1$, natural $m \geq 2$ and arbitrary measurable subset $E \subset[0,1]$ such that $\mu(E)=1-1 / M^{2}$ there exists a complete orthonormal system $\Psi$ on $[0,1]$ uniformly bounded by $M$ such that the multiple Fourier series of any integrable function $f \in L\left([0,1]^{m}\right)$ with respect to the product system $\Psi(m)$ converges cubically to $f$ a.e. on $E^{m}$.

$\lambda$-restricted convergence is more general than cubical convergence. For $\mathbf{k} \in \mathbb{N}^{m}$ and $\lambda \geq 1$ we consider the $\lambda$-restricted partial sums

$$
S_{\mathbf{k}}^{\Phi}(f)\left(x_{1}, \ldots, x_{m}\right)=\sum_{\mathbf{n}=\mathbf{1}}^{\mathbf{k}} a_{\mathbf{n}}(f) \varphi_{\mathbf{n}}(\mathbf{x}) \equiv \sum_{n_{1}=1}^{k_{1}} \ldots \sum_{n_{m}=1}^{k_{m}} a_{\mathbf{n}}(f) \varphi_{\mathbf{n}}(\mathbf{x}),
$$

where

$$
\frac{1}{\lambda} \leq \frac{k_{i}}{k_{j}} \leq \lambda \quad \text { for any } 1 \leq i, j \leq m .
$$

If the partial sums (4) converge in some sense (pointwise on a given set, in measure, in some metric) as $|\mathbf{k}| \rightarrow \infty$, where $\mathbf{k}$ satisfies (5), then the series (1) is said to converge $\lambda$-restrictively in the same sense. Otherwise, it diverges $\lambda$-restrictively in the relevant sense.

In the present paper we prove the following

Theorem 1. For any $M>1$, natural $m \geq 2$ and arbitrary measurable subset $E \subset[0,1]$ such that $\mu(E)=1-1 / M^{2}$ there exists a complete orthonormal system $\Psi$ on $[0,1]$, uniformly bounded by $M$, such that the multiple Fourier series of any integrable function $f \in L\left([0,1]^{m}\right)$ with respect to the product system $\Psi(m) \lambda$-restrictively converges to $f$ a.e. on $E^{m}$ for any $\lambda \geq 1$.

In the proof we use the system constructed in [3].

The study of the so-called spherical partial sums

$$
S_{R}(f)(\mathbf{x})=\sum_{|\mathbf{n}| \leq R} a_{\mathbf{n}}(f) \varphi_{\mathbf{n}}(\mathbf{x})
$$

where $R>1$, usually leads to results that in many cases are surprisingly different from the results for cubic partial sums.

In the present paper we give sharp estimates for the sets of divergence for some general methods of summation which of course include the spherical convergence.

Let $G \subset[0,1]^{m}$ be a closed set that contains $[0, \delta]^{m}$ for some $\delta>0$. For $R>1$ define 


$$
{ }^{R} G=\left\{\mathbf{x} \in[0, \infty)^{m}: \frac{1}{R} \mathbf{x} \in G\right\}, \quad S_{G, R}^{\Phi}(f)(\mathbf{x})=\sum_{\mathbf{n} \in R_{G}} a_{\mathbf{n}}(f) \varphi_{\mathbf{n}}(\mathbf{x}) .
$$

If $\lim _{R \rightarrow \infty} S_{G, R}^{\Phi}(f)(\mathbf{x})$ exists in some sense, we will say that the series (1) $G$-converges in the same sense. Otherwise, it $G$-diverges in the indicated sense.

Let $m \geq 2$. Denote by $\mathbf{A}(m)$ the class of closed convex sets in $[0,1]^{m}$ such that

1) $\mu_{m}(A)>0$ for all $A \in \mathbf{A}(m)$;

2) if $\mathbf{x} \in A \in \mathbf{A}(m)$ then $\prod_{j=1}^{m}\left[0, x_{j}\right] \subseteq A$.

The following two theorems hold.

THEOREM 2. Let $\Phi=\left\{\varphi_{n}(x)\right\}_{n=1}^{\infty}$ be a complete orthonormal system on $[0,1]$ which satisfies $(3)$, let $m \geq 2$ and let $\Phi(m)$ be the corresponding product system. Then for any $A \in \mathbf{A}(m)$ there exists a function $f(\mathbf{x}) \in L\left([0,1]^{m}\right)$ whose Fourier series with respect to $\Phi(m)$ A-diverges everywhere on some measurable set $\mathcal{H} \subset[0,1]^{m}$ with $\mu_{m}(\mathcal{H}) \geq 1-\left(1-1 / M^{2}\right)^{m}$.

TheOREM 3. Let $\Phi=\left\{\varphi_{n}(x)\right\}_{n=1}^{\infty}$ be an orthonormal system on $[0,1]$ which satisfies (3), let $m \geq 2$ and let $\Phi(m)$ be the corresponding product system. Then for any $A \in \mathbf{A}(m)$ there exists a function $f(\mathbf{x}) \in L\left([0,1]^{m}\right)$ whose Fourier series with respect to $\Phi(m)$ A-diverges everywhere on some measurable set $\mathcal{H}_{0} \subset[0,1]^{m}$ with $\mu_{m}\left(\mathcal{H}_{0}\right) \geq 1 / M^{2 m}$.

The example constructed above shows the sharpness of the estimate in Theorem 3. The sharpness of Theorem 2 is proved for sets that belong to $\mathbf{A}(m)$ under an additional condition.

We will use the following notation. If $A \in \mathbf{A}(m)$ then for $j=1, \ldots, m$ we put

$$
\begin{aligned}
\alpha_{j} & =\alpha_{j}(A)=\sup _{\mathbf{x} \in A} x_{j}>0, \quad \alpha(A)=\max _{1 \leq j \leq m} \alpha_{j}(A), \\
\beta & =\beta(A)=\max _{1 \leq i, j \leq m} \frac{\alpha_{i}}{\alpha_{j}} .
\end{aligned}
$$

Let $\theta=\left\{j_{1}, \ldots, j_{q}\right\} \subset Z_{m}=\{1, \ldots, m\}$ be a nonempty set such that $\bar{\theta}=Z_{m} \backslash \theta=\left\{i_{1}, \ldots, i_{p}\right\} \neq \emptyset$. Then for every $\mathbf{x} \in \mathbb{R}^{m}$ we write $\mathbf{x}_{\theta}=$ $\left(x_{j_{1}}, \ldots, x_{j_{q}}\right)$ and $\mathbf{x}_{\bar{\theta}}=\left(x_{i_{1}}, \ldots, x_{i_{p}}\right)$.

The following obvious proposition explains the choice of the class $\mathbf{A}(m)$.

Proposition 1. Let $A \in \mathbf{A}(m)$ and $\theta=\left\{j_{1}, \ldots, j_{q}\right\} \subset Z_{m}$ with $\bar{\theta}=$ $Z_{m} \backslash \theta=\left\{i_{1}, \ldots, i_{p}\right\} \neq \emptyset$ and let $\mathbf{y} \in \mathbb{R}^{m}$ be such that $0 \leq y_{j_{s}}<\alpha_{j_{s}}$ for all $1 \leq s \leq q$. Then the set

$$
A(\theta, \mathbf{y})=\left\{\mathbf{x}_{\bar{\theta}} \in[0,1]^{p}: \mathbf{x} \in A \text { and } x_{l}=y_{l} \text { for } l \in \theta\right\}
$$

belongs to the class $\mathbf{A}(m-\operatorname{card} \theta)$. 
In order to define a subclass of $\mathbf{A}(m)$ which we will use to obtain results of positive type, we denote by $\mathbf{A}(m, d), d \geq 1$, the subset of all sets $A \in \mathbf{A}(m)$ such that $\beta(A(\theta, \mathbf{y})) \leq d$ for all $\theta=\left\{j_{1}, \ldots, j_{q}\right\} \subset Z_{m}$ with $Z_{m} \backslash \theta \neq \emptyset$ and all $\mathbf{y} \in \mathbb{R}^{m}$, with $0 \leq y_{j_{s}}<\alpha_{j_{s}}$ for $1 \leq s \leq q$.

For example, the unit sphere in $\mathbb{R}^{m}$ belongs to $\mathbf{A}(m, 1)$ for every $m \geq 2$.

We set

$$
\mathbf{A}^{\prime}(m)=\bigcup_{d \geq 1} \mathbf{A}(m, d) .
$$

Remark 1. It is easy to check that $\mathbf{A}^{\prime}(2)=\mathbf{A}(2)$ and $\mathbf{A}^{\prime}(m) \neq \mathbf{A}(m)$ for $m \geq 3$.

Indeed, for $m=3$ one can easily verify that the set

$$
A=\{(x, y, z): 0 \leq z \leq 1, x, y \geq 0, x+y \leq 1\} \in \mathbf{A}(3)
$$

but $A \notin \mathbf{A}^{\prime}(3)$. When $m>3$ we take $A \times[0,1]^{m-3}$ instead of $A$.

The following result holds.

THEOREM 4. For any $M>1$, natural $m \geq 2$ and arbitrary measurable subset $E \subset[0,1]$ such that $\mu(E)=1-1 / M^{2}$, there exists a complete orthonormal system $\Psi$ on $[0,1]$, uniformly bounded by $M$, such that the multiple Fourier series of any integrable function $f \in L\left([0,1]^{m}\right)$ with respect to the product system $\Psi(m) A$-converges to $f$ a.e. on the set $E^{m}$ for any $A \in \mathbf{A}^{\prime}(m)$.

We would like to indicate the differences that appear in the description of sets of convergence for the one-dimensional and multi-dimensional cases. First let us define precisely what we mean by a set of convergence.

Definition. Let $\Phi=\left\{\varphi_{n}\right\}_{n=1}^{\infty}$ be an orthonormal system on $[0,1]$, $m \geq 2$ and $G \subset[0,1]^{m}$ a closed set. We will say that a measurable set $\mathcal{E} \subset[0,1]^{m}$ is a set of $G$-convergence (resp. $\lambda$-restricted convergence) for the product system $\Phi(m)$ if for any integrable function $f \in L\left([0,1]^{m}\right)$ the multiple Fourier series of $f$ with respect to the system $\Phi(m) G$-converges (resp. $\lambda$-restrictively converges) almost everywhere on the set $\mathcal{E}$.

In the one-dimensional case, according to Theorem B, any set of measure $1-1 / M^{2}$ can be a set of convergence for some CONS uniformly bounded by $M$.

By Theorem A we easily obtain the following assertion about sets of $G$-convergence and $\lambda$-restricted convergence.

REMARK 2. Let $G \subset[0,1]^{m}$ be a convex set. If a measurable set $\mathcal{E} \subset$ $[0,1]^{m}$ is a set of $G$-convergence (resp. $\lambda$-restricted convergence) for the product system $\Phi(m)$, where $\Phi=\left\{\varphi_{n}\right\}_{n=1}^{\infty}$ is an orthonormal system on [0,1] that satisfies (3), then the measure of the projection of $\mathcal{E}$ on any coordinate axis is less than or equal to $1-1 / M^{2}$. 
In particular, we have the following concrete result.

COROLlary 1. For every $m \geq 2$ and for every $\varepsilon>0$ there exists a set $\mathcal{E} \subset[0,1]^{m}$, with $\mu(\mathcal{E})=\varepsilon$, such that for every uniformly bounded complete orthonormal system $\Phi$ and for every convex set $G \subset[0,1]^{m}$, there exists a function $f \in L\left([0,1]^{m}\right)$ whose Fourier series with respect to the product system $\Phi(m) G$-diverges (resp. $\lambda$-restrictively diverges) on some measurable set $\mathcal{E}_{1} \subset \mathcal{E}$ with $\mu\left(\mathcal{E}_{1}\right)>0$.

3. Divergence. In this section we prove Theorems 2 and 3. For this purpose we need a variant of the Saks Resonance Theorem (cf. [2]). Denote by $L_{X}^{0}(d \nu)$ the space of measurable $\nu$-almost everywhere finite functions defined on $X$ with the quasi-norm

$$
\|f\|_{L_{X}^{0}(d \nu)}:=\int_{X} \frac{|f(t)|}{1+|f(t)|} d \nu(t) .
$$

Theorem F. Let $X=[0,1]^{m}$ and $S_{n}$ be a sequence of continuous linear operators from $L_{X}\left(d \mu_{m}\right)$ to $L_{X}^{0}\left(d \mu_{m}\right)$. Then there exists a measurable set $Y \subset X$ such that for all $f \in L\left([0,1]^{m}\right)$,

$$
\mu\left(\left\{t \in Y: \sup _{n \in \mathbb{N}}\left|S_{n}(f)(t)\right|<\infty\right\}\right)=\mu(Y)
$$

and also

$$
\mu\left(\left\{t \in X \backslash Y: \sup _{n \in \mathbb{N}}\left|S_{n}(f)(t)\right|=\infty\right\}\right)=\mu(X \backslash Y)
$$

except for $f \in F^{1}$, where $F^{1}$ is a set of the first category in $L\left([0,1]^{m}\right)$.

The following simple result will be useful in the proofs of Theorems 2 and 3 .

Lemma 1. Let $A \in \mathbf{A}(m)$ and $\nu$ be a natural number. Then for any $N>\frac{\beta(A)}{\alpha_{1}(A)}(m-1) \nu$ we have

$$
\left[0, \alpha_{1}(A) \cdot N-(m-1) \beta(A) \nu\right] \times[0, \nu]^{m-1} \subset{ }^{N} A .
$$

Proof. According to the definition of $\beta(A)$ we have

$$
(\underbrace{0, \ldots, 0}_{j-1}, \alpha_{1}(A) / \beta(A), \underbrace{0, \ldots, 0}_{m-j}) \in A \quad \text { for every } j \in[2, m] .
$$

Since $A$ is convex we see that for any $j \in[2, m]$,

$$
(\alpha_{1}(A)-\frac{1}{N}(m-1) \beta(A) \nu, \underbrace{0, \ldots, 0}_{j-2}, \frac{1}{N}(m-1) \nu, \underbrace{0, \ldots, 0}_{m-j}) \in A .
$$


Again using the convexity of $A$ we obtain

$$
\left(\alpha_{1}(A)-\frac{1}{N}(m-1) \beta(A) \nu, \frac{1}{N} \nu, \ldots, \frac{1}{N} \nu\right) \in A .
$$

Hence, since $A \in \mathbf{A}(m)$, we obtain (7).

Proof of Theorem 2. Note that since $\chi_{[0,1]} \in L_{2}([0,1])$, the series

$$
\sum_{n=1}^{\infty} c_{n} \varphi_{n}(x), \quad \text { where } \quad c_{n}=\int_{0}^{1} \varphi_{n}(t) d t
$$

converges in the $L^{2}$-metric and consequently in measure to $\chi_{[0,1]}$. Hence, some sequence of partial sums of the series converges a.e. on $[0,1]$ to $\chi_{[0,1]}$. This means that for a given $\gamma>0$ there exists a polynomial

$$
Q(t)=\sum_{n=1}^{\nu} c_{n} \varphi_{n}(t)>\frac{1}{2}
$$

for

$$
t \in F_{\gamma} \subset[0,1], \quad \text { where } \mu\left(F_{\gamma}\right)>1-\gamma .
$$

By Theorem A we find a measurable subset $E \subset[0,1]$ and a function $g \in L([0,1])$ such that

$$
\mu(E) \geq 1 / M^{2}
$$

and the Fourier series of $g$ with respect to the system $\Phi$ unboundedly diverges on $E$.

For any $l(1 \leq l \leq m)$ we define a function of $m$ variables

$$
f_{l}(\mathbf{x})=f_{l}\left(x_{1}, \ldots, x_{m}\right)=g\left(x_{l}\right) \prod_{j \neq l}^{m} Q\left(x_{j}\right) .
$$

Evidently, $f_{l} \in L\left([0,1]^{m}\right)$ for all $l(1 \leq l \leq m)$. The conditions imposed on the sets that belong to the class $\mathbf{A}(m)$ are symmetric with respect to the axes, thus the assertion of Lemma 1 remains true if in the formulation we take any coordinate $l(1 \leq l \leq m)$ instead of the first coordinate. For any $N>\frac{\beta(A)}{\alpha_{l}(A)}(m-1) \nu$ we can write

$$
S_{N}^{A}\left(f_{l}\right)(\mathbf{x})=S_{n}(g)\left(x_{l}\right) \prod_{j \neq l}^{m} Q\left(x_{j}\right)+F\left(x_{1}, \ldots, x_{m}\right),
$$

where $n$ is the greatest natural number for which

$$
{ }^{N} A \supseteq \mathcal{A}_{n}^{\nu}=\underbrace{[0, \nu] \times \ldots \times[0, \nu]}_{l-1} \times[0, n] \times \underbrace{[0, \nu] \times \ldots \times[0, \nu]}_{m-l} .
$$

Let $\mathcal{B}_{n}^{\nu}=\left\{\mathbf{k} \in \mathcal{A}_{n}^{\nu}: k_{l}>n\right.$ and $0 \leq k_{j} \leq \nu$ for $\left.j \neq l\right\}$. 
By the above observation about Lemma 1 we have $n>\alpha_{l}(A) \cdot N$ $-(m-1) \beta(A) \nu-1$. Hence, for the function $F$ defined in (10) we get the estimate

$$
\begin{aligned}
\|F\|_{\infty} & \leq \sum_{\mathbf{k} \in \mathcal{B}_{n}^{\nu}}\left|a_{\mathbf{k}}(f)\right|\left\|\varphi_{\mathbf{k}}\right\|_{\infty} \leq(m-1) \beta(A) \nu^{m} M^{2 m}\|f\|_{1} \\
& \leq(m-1) \beta(A) \nu^{m} M^{2 m}\|g\|_{1} .
\end{aligned}
$$

Hence the Fourier series of $f_{l}(\mathbf{x})$ with respect to $\Phi(m)$ unboundedly $A$ diverges on

$$
\Theta_{F_{\gamma}}^{l}(E)=\left\{\left(x_{1}, \ldots, x_{m}\right) \in[0,1]^{m}: x_{i} \in F_{\gamma}, i \neq l \text {, and } x_{l} \in E\right\} .
$$

By (9) and Theorem $\mathrm{F}$, for any any $l(1 \leq l \leq m)$ there exists a function $h_{l} \in L\left([0,1]^{m}\right)$ whose Fourier series with respect to $\Phi(m)$ unboundedly $A$-diverges a.e. on the set

$$
H_{l}=\Theta^{l}(E)=\left\{\left(x_{1}, \ldots, x_{m}\right) \in[0,1]^{m}: x_{i} \in[0,1], i \neq l \text {, and } x_{l} \in E\right\} .
$$

Another application of Theorem F yields a function $f \in L\left([0,1]^{m}\right)$ whose Fourier series with respect to $\Phi(m)$ unboundedly $A$-diverges a.e. on the set

$$
\mathcal{H}=\bigcup_{l=1}^{m} H_{l}=\bigcup_{l=1}^{m} \Theta^{l}(E) .
$$

To finish the proof of Theorem 2 we only need to check that

$$
\mu_{m}\left([0,1]^{m} \backslash \mathcal{H}\right)=\mu_{m}\left([0,1]^{m} \backslash \bigcup_{l=1}^{m} H_{l}\right) \leq\left(1-\frac{1}{M^{2}}\right)^{m} .
$$

Hence

$$
\mu_{m}(\mathcal{H}) \geq 1-\left(1-\frac{1}{M^{2}}\right)^{m} .
$$

Proof of Theorem 3. The proof is similar to the proof of Theorem 2. Let $g$ and $E$ be as in the latter proof. Now we define a function of $m$ variables

$$
f_{0}(\mathbf{x})=f_{0}\left(x_{1}, \ldots, x_{m}\right)=g\left(x_{1}\right) \prod_{j \geq 2}^{m} \varphi_{1}\left(x_{j}\right) .
$$

Let

$$
F_{r}^{*}=\left\{t \in[0,1]:\left|\varphi_{1}(t)\right| \geq 2^{-r / 2 m}\right\} .
$$

Then it follows that

$$
\begin{aligned}
1 & =\int_{[0,1]}\left|\varphi_{1}(t)\right|^{2} d t=\int_{F_{r}^{*}}\left|\varphi_{1}(t)\right|^{2} d t+\int_{[0,1] \backslash F_{r}^{*}}\left|\varphi_{1}(t)\right|^{2} d t \\
& \leq M^{2} \mu\left(F_{r}^{*}\right)+2^{-r / m} .
\end{aligned}
$$


Hence, $\liminf _{r \rightarrow \infty} \mu\left(F_{r}^{*}\right) \geq 1 / M^{2}$ and

$$
\mu\left(F^{*}\right) \geq 1 / M^{2}, \quad \text { where } \quad F^{*}=\limsup _{r \rightarrow \infty} F_{r}^{*} .
$$

The same arguments as in the proof of Theorem 2 show that there exists a function $f \in L\left([0,1]^{m}\right)$ whose Fourier series with respect to $\Phi(m)$ unboundedly $A$-diverges on the set

$$
\mathcal{H}_{0}=\Theta_{F^{*}}^{1}(E)=\left\{\left(x_{1}, \ldots, x_{m}\right) \in[0,1]^{m}: x_{i} \in F^{*}, i \geq 2, \text { and } x_{1} \in E\right\} .
$$

Evidently, $\mu_{m}\left(\mathcal{H}_{0}\right) \geq 1 / M^{2 m}$.

\section{Convergence}

Proof of Theorem 1. Without loss of generality we may assume that $E=\left[0,1-1 / M^{2}\right]$, since in general using the function $\xi(x)=\mu((0, x) \cap E)$ one can establish a measure-preserving mapping of $E$ onto the segment $[0, \mu(E)]$. For simplicity of notation we suppose $M=\sqrt{2}$.

Under the above assumptions we can show that the system $\left\{\eta_{n}\right\}_{n=1}^{\infty}$ constructed in [3] can serve as an example which proves the assertion of Theorem 1. One only needs to use the fact that the $m$-fold Fourier-Haar series $\lambda$-restrictively converges almost everywhere and to repeat the proof of Theorem $\mathrm{E}$ with simple modifications.

But there is also another way to prove Theorem 1, which seems simpler. We can define a permutation of the system $\left\{\eta_{n}\right\}_{n=1}^{\infty}$ such that for the resulting system $\Psi, \lambda$-restrictive convergence of the multiple Fourier series of any integrable function with respect to $\Psi(m)$ follows from the cubical convergence of the series with respect to the product system of $\left\{\eta_{n}\right\}_{n=1}^{\infty}$.

We are going to use the same idea in the proof of Theorem 4. To avoid repetition we will give here only a vague idea of the proof. For the reader who is going to read the proof of Theorem 4 it will be useful to get acquainted with the idea of the proof in a geometrically simpler case.

To present that idea we recall the following property of the system $\left\{\eta_{n}\right\}_{n=1}^{\infty}$ (see [3]): there exist a sequence of measurable sets

$$
A_{1} \subset A_{2} \subset \ldots, \quad \bigcup_{r=1}^{\infty} A_{r}=[0,1 / 2),
$$

and an infinite subsystem $\Phi_{1}=\left\{\eta_{\nu_{k}}\right\}_{k=1}^{\infty}, \nu_{1}<\nu_{2}<\ldots$, of $\left\{\eta_{n}\right\}_{n=1}^{\infty}$ such that for every $r$ there is $N_{r}$ for which

$$
\eta_{\nu_{k}}(x)=0 \quad \text { for all } x \in A_{r} \text { and } k \geq N_{r} .
$$

Let $\left\{\mu_{k}\right\}_{k=1}^{\infty}$ be the increasing subsequence of natural numbers such that

$$
\begin{aligned}
& \left\{\mu_{k}\right\}_{k=1}^{\infty} \cup\left\{\nu_{k}\right\}_{k=1}^{\infty}=\mathbb{N}, \\
& \left\{\mu_{k}\right\}_{k=1}^{\infty} \cap\left\{\nu_{k}\right\}_{k=1}^{\infty}=\emptyset
\end{aligned}
$$


Let

$$
\psi_{1}=\eta_{\nu_{1}}, \quad \psi_{2^{k^{2}}}=\eta_{\mu_{k}} \quad \text { for } k=1,2, \ldots,
$$

and for any $j \in \mathbb{N}$ with $2^{k^{2}}<j<2^{(k+1)^{2}} k=1,2, \ldots$, put

$$
\psi_{j}=\eta_{\nu_{j-k}} .
$$

According to the construction of the system $\Psi$, for any $\lambda \geq 1$ and $n \in \mathbb{N}$ we can find $N=N(\lambda, n)$ such that for any $\mathbf{k}=\left(k_{1}, \ldots, k_{m}\right)$ that satisfies (5) and $\min _{1 \leq i \leq m} k_{i} \geq N$, the partial sum

$$
\sum_{n_{1}=1}^{k_{1}} \ldots \sum_{n_{m}=1}^{k_{m}} a_{\mathbf{n}}(f) \psi_{\mathbf{n}}(\mathbf{x})
$$

is equal on $A_{n}$ to some cubic partial sum of the Fourier series of $f$ with respect to the $m$-fold product system of $\left\{\eta_{n}\right\}_{n=1}^{\infty}$ plus a number, depending only on $m$, of additional terms, which can be estimated by maximal functions using the inequalities for cubic partial sums established in [1].

Now we turn to the proof of Theorem 4, where this idea will be realized in a more complicated situation.

Proof of Theorem 4. Again as in the proof of Theorem 1, without loss of generality, we may assume that $E=\left[0,1-1 / M^{2}\right]$ and $M=\sqrt{2}$. We also assume that $\alpha(A)=1$.

First of all we point out all those properties of $\left\{\eta_{n}\right\}_{n=1}^{\infty}$ which will allow us to prove Theorem 4.

We will use some properties of the Haar system. The Haar functions may be defined in the following manner: For all $t \in[0,1]$, let $h_{1}(t)=1$, and for $k=0,1,2, \ldots$ and $j=1,2, \ldots, 2^{k}$, let

$$
h_{k}^{(j)}(t)= \begin{cases}2^{k / 2} & \text { if }(2 j-2) / 2^{k+1}<t<(2 j-1) / 2^{k+1}, \\ -2^{k / 2} & \text { if }(2 j-1) / 2^{k+1}<t<2 j / 2^{k+1} \\ 0 & \text { otherwise, }\end{cases}
$$

and for $n=2^{k}+j$, let $h_{n}=h_{k}^{(j)}$.

The support of the Haar function $h_{n}$ will be denoted by $\Delta_{n}$ or $\Delta_{k}^{(j)}$.

Let

$$
\begin{aligned}
& \bar{h}_{n}(x)= \begin{cases}\sqrt{2} h_{n}(2 x) & \text { if } x \in(0,1 / 2), \\
0 & \text { if } x \in(1 / 2,1],\end{cases} \\
& \overline{\bar{h}}_{n}(x)= \begin{cases}0 & \text { if } x \in[0,1 / 2], \\
\sqrt{2} h_{n}(2 x-1) & \text { if } x \in(1 / 2,1] .\end{cases}
\end{aligned}
$$

The system $\left\{\eta_{n}\right\}_{n=1}^{\infty}$ is constructed so that for a certain increasing sequence $\left\{l_{j}\right\}_{j=0}^{\infty}$ of numbers with $l_{0}=1$ there exist numbers $1 \leq \kappa_{n}<2^{n}$ 
such that

$$
\lim _{n \rightarrow \infty} \frac{\kappa_{n}}{2^{n}}=1
$$

and for $n=2,3, \ldots$ and $1 \leq j \leq \kappa_{n}, 1 \leq i \leq 2^{2 n}, 1 \leq \nu \leq 2^{n+3}$,

$$
\eta_{l_{n-1}+(j-1) 2^{3 n+3}+(i-1) 2^{n+3}+\nu}(x)=2^{-n} 2^{-(n+3) / 2} \bar{h}_{n}^{(j)}(x) \quad \text { if } x \in A_{n},
$$

where

$$
A_{n}=\bigcup_{l=1}^{\kappa_{n}} \Delta_{n}^{(l)} .
$$

Moreover for any $x \in(0,1 / 2)$ there exists $n_{x} \in \mathbb{N}$ such that for all $\phi \in L[0,1]$ and $n \geq n_{x}$,

$$
S_{l_{n}}(\phi, x)=\sum_{k=1}^{l_{n}} a_{k}(\phi) \eta_{k}(x)=\sum_{i=1}^{2^{n}} b_{i}(\phi) \bar{h}_{i}(x),
$$

where

$$
a_{k}(\phi)=\int_{0}^{1} \phi(t) \eta_{k}(t) d t, \quad b_{i}(\phi)=\int_{0}^{1} \phi(t) \bar{h}_{i}(t) d t
$$

If we put

$$
k_{n}(j)=l_{n-1}+(j-1) 2^{3 n+3}
$$

then for all $n=2,3, \ldots$ and $1 \leq j \leq \kappa_{n}$,

$$
\sum_{k=1}^{k_{n}(j+1)} a_{k}(f) \eta_{k}(x)=\sum_{l=1}^{2^{n-1}} b_{l}(f) \bar{h}_{l}(x)+\sum_{i=1}^{j} b_{i}^{n}(f) \bar{h}_{i}^{(n)}(x) \quad \text { if } x \in A_{n} .
$$

One has to observe that

$$
\eta_{k}(x)=0 \quad \text { if } x \in A_{n} \text { and } k_{n}\left(\kappa_{n}\right)<k \leq l_{n} .
$$

Let $S_{N}(f, \mathbf{x})$ be the cubic partial sums of the Fourier series of $f \in$ $L\left([0,1]^{m}\right)$ with respect to the $m$-fold product system of $\left\{\eta_{n}\right\}_{n=1}^{\infty}$. If we set $\mathbf{l}=(l, \ldots, l) \in \mathbb{N}^{m}$, where $k_{n}\left(\kappa_{n}\right)<l \leq l_{n}$, then

$$
S_{\mathbf{l}}(f, \mathbf{x})=\int_{[0,1]^{m}} f(\mathbf{t}) \prod_{p=1}^{m} D_{l}\left(x_{p}, t_{p}\right) d t_{1} \ldots d t_{m}
$$

where

$$
D_{l}(x, t)=\sum_{k=1}^{l} \eta_{k}(x) \eta_{k}(t)
$$

By Schauder's lemma about the partial sums of Fourier-Haar series and (17), (18) we obtain

$$
\left|S_{\mathbf{k}_{n}(j)}(f, \mathbf{x})\right| \leq M_{m}(f, \mathbf{x}), \quad \mathbf{x} \in A_{n}^{m},
$$


where $\mathbf{k}_{n}(j)=\left(k_{n}(j), \ldots, k_{n}(j)\right)$, and $M_{m}(f, \mathbf{x})$ is the standard maximal function taken over cubes with sides parallel to the axes in $[0,1]^{k}$.

We notice that $A_{n} \subset A_{n+1} \subset \ldots \rightarrow(0,1 / 2)$, off a denumerable set.

Thus we have to take care of the partial sums $S_{\mathbf{l}}(f, \mathbf{x})$, where $\mathbf{l}=(l, \ldots, l)$ and $l_{n-1}<l \leq k_{n}\left(\kappa_{n}\right)$. So we can suppose in our further deduction that the numbers $n$ and $j$ are fixed; for simplicity we suppress them in the notation. Let $\Upsilon=\operatorname{supp} \bar{h}_{n}^{(j)}, \Upsilon^{*}=\operatorname{supp} \overline{\bar{h}}_{n}^{(j)}$, and for any $x \in A_{n}, x \notin \Upsilon$ denote by $\Delta(x)$ the largest dyadic interval where all the functions on the right side of (17) are constant and $x \in \Delta(x)$.

The partial sums of the Fourier series of a function $\phi$ with respect to the system $\left\{\eta_{n}\right\}_{n=1}^{\infty}$ also have the following property: for all $n=2,3, \ldots$ and $1 \leq j \leq \kappa_{n}, 1 \leq i \leq 2^{2 n}$,

$$
S_{k_{n}(j)+i 2^{2 n}}(\phi, x)-S_{k_{n}(j)+(i-1) 2^{2 n}}(\phi, x)=\int_{0}^{1} \phi(t) g_{j i}^{(n)}(t) d t g_{j i}^{(n)}(x),
$$

where

$$
g_{j i}^{(n)}(x)=2^{-n} \bar{h}_{n}^{(j)}(x)+2^{-n} \sum_{l=2}^{2^{2 n}} h_{l}\left(i 2^{-n}-2^{-n-1}\right) \overline{\bar{h}}_{n}^{(j)}(x) r_{N_{n}+l}(x),
$$

where $r_{n}(t)=\operatorname{sgn}\left(\sin 2^{n} \pi t\right), n \in \mathbb{N}$, are the Rademacher functions and $N_{n}$ is some natural number. It was shown in [4] that for $x \in A_{n}$,

$$
\left|\sum_{i=1}^{l} \int_{0}^{1} \phi(t) g_{j i}^{(n)}(t) d t g_{j i}^{(n)}(x)\right| \leq 6\left(\mu\left(\Upsilon^{*}\right)\right)^{-1} \int_{\Upsilon^{*}}|\phi(t)| d t \cdot \chi_{\Upsilon}(x) .
$$

Thus

$$
\left|S_{k_{n}(j)+i 2^{2 n}}(\phi, x)-S_{k_{n}(j)}(\phi, x)\right| \leq 6\left(\mu\left(\Upsilon^{*}\right)\right)^{-1} \int_{\Upsilon^{*}}|\phi(t)| d t \cdot \chi_{\Upsilon}(x) .
$$

Finally, in [4] it was also shown that for all $n=2,3, \ldots$ and $1 \leq j \leq \kappa_{n}$, $1 \leq i \leq 2^{2 n}, 1 \leq \nu \leq 2^{n+3}$, and all $x \in A_{n}$,

$$
\begin{aligned}
& \left|S_{k_{n}(j)+(i-1) i 2^{2 n}+\nu}(\phi, x)-S_{k_{n}(j)+(i-1) 2^{2 n}}(\phi, x)\right| \\
& \leq 2^{-3 n / 2+1} \int_{0}^{1 / 2}\left|\phi(t) \bar{h}_{n}^{(j)}(t)\right| d t+6 \int_{1 / 2}^{1}|\phi(t)| d t \leq 6\|\phi\|_{1} .
\end{aligned}
$$

Using (20), (21) and (17) we find that for all $l\left(k_{n}(j)<l \leq k_{n}(j+1)\right)$,

$$
S_{l}(\phi, x)=\lambda_{l}(x) \quad \text { if } x \in \Upsilon \text {, }
$$

where

$$
\frac{1}{6}\left|\lambda_{l}(x)\right| \leq\left(\frac{1}{\mu(\Upsilon)} \int_{\Upsilon}|\phi(t)| d t+\frac{1}{\mu\left(\Upsilon^{*}\right)} \int_{\Upsilon^{*}}|\phi(t)| d t+\|\phi\|_{1}\right) \chi_{\Upsilon}(x),
$$


and

$$
S_{l}(\phi, x)=\frac{1}{\mu(\Delta(x))} \int_{\Delta(x)} \phi(t) d t \quad \text { if } x \in A_{n} \text { and } x \notin \Upsilon .
$$

The last formula follows immediately from (17) upon recalling Schauder's lemma about the partial sums of Fourier-Haar series. From (24) we deduce that if $\mathbf{x} \in A_{n}^{m}$ and $x_{\nu} \notin \Upsilon$ for all $1 \leq \nu \leq m$, then

$$
S_{\mathbf{l}}(f, \mathbf{x})=\frac{1}{\mu_{m}(\Delta(\mathbf{x}))} \int_{\Delta(\mathbf{x})} f(\mathbf{t}) d \mathbf{t},
$$

where $\mathbf{x}=\left(x_{1}, \ldots, x_{m}\right)$ and $\Delta(\mathbf{x})=\prod_{p=1}^{m} \Delta\left(x_{p}\right)$.

Let us see what happens if for some indices $\theta=\left\{j_{\nu}\right\}_{\nu=1}^{q}$ with $q<m$ we have $x_{j_{\nu}} \in \Upsilon$ and $x_{i} \notin \Upsilon$ for $i \neq j_{\nu}, 1 \leq \nu \leq q$. Recall the notation $\bar{\theta}=Z_{m} \backslash \theta=\left\{i_{1}, \ldots, i_{p}\right\} \neq \emptyset$ and

$$
\Delta\left(\mathbf{x}_{\bar{\theta}}\right)=\prod_{\nu=1}^{m-q} \Delta\left(x_{i_{\nu}}\right)
$$

and

$$
f_{\theta}\left(\mathbf{t}_{\theta}, \mathbf{x}_{\bar{\theta}}\right)=\frac{1}{\mu_{m-q}\left(\Delta\left(\mathbf{x}_{\bar{\theta}}\right)\right)} \int_{\Delta\left(\mathbf{x}_{\bar{\theta}}\right)} f(\mathbf{t}) d \mathbf{t}_{\bar{\theta}},
$$

where $d \mathbf{t}_{\bar{\theta}}=d t_{i_{1}} \ldots d t_{i_{p}}$.

In [1] we have proved that

$$
\left|S_{\mathbf{l}}(f, \mathbf{x})\right| \leq 12^{m} \sum_{\omega} \sum_{\sigma} M_{m-c_{\omega}}\left(f_{\theta} ; \mathbf{y}_{\omega^{\prime}}, \mathbf{x}_{\bar{\theta}}\right)
$$

where $M_{k}(F, u)$ is the standard maximal function of $F$ taken over cubes with sides parallel to the axes in $[0,1]^{k}$; the first sum is taken over all possible subsets $\omega \subset \theta, c_{\omega}=\operatorname{card} \omega, \omega^{\prime}=\theta \backslash \omega$; the second sum is taken over all possible 0-1 vectors in $\mathbb{R}^{c_{\omega^{\prime}}}, \mathbf{t}_{\omega^{\prime}}=\left(t_{j_{\nu_{1}}}, \ldots, t_{j_{\nu_{c^{\prime}}}}\right)$, where $\omega^{\prime}=\left(j_{\nu_{1}}, \ldots, j_{\nu_{c^{\prime}}}\right)$; and also $\Delta_{\omega^{\prime} \sigma}(\mathbf{x})=\prod_{p=1}^{c_{\omega^{\prime}}} \Upsilon_{\sigma_{p}}$, where $\Upsilon_{\sigma_{p}} \stackrel{=}{=}$ if $\sigma_{p}=0$ and $\Upsilon_{\sigma_{p}}=\Upsilon^{*}$ if $\sigma_{p}=1, \sigma=\left(\sigma_{1}, \ldots, \sigma_{c_{\omega^{\prime}}}\right)$; and $y_{j_{\nu_{i}}}=x_{\nu_{i}}$ if $\sigma_{\nu_{i}}=0$ and $y_{j_{\nu_{i}}}=x_{j_{\nu_{i}}}+1 / 2$ if $\sigma_{\nu_{i}}=1$.

If $\mathbf{x} \in \Upsilon^{m}$, as above we obtain an estimate similar to (27).

Now, in order to construct the rearrangement of the system $\left\{\eta_{n}\right\}_{n=1}^{\infty}$ which will satisfy the conditions of Theorem 4 we divide it into two parts: $\Phi_{1}=\left\{\eta_{n_{k}}(x)\right\}_{k=1}^{\infty}, n_{1}<n_{2}<\ldots$, where $\left\{n_{k}\right\}_{k=1}^{\infty}=\bigcup_{n=2}^{\infty}\left\{i \in \mathbb{N}: l_{n-1}<\right.$ $\left.i \leq k_{n}\left(\kappa_{n}\right)\right\}$, and $\Phi_{2}=\left\{\eta_{m_{k}}\right\}_{k=1}^{\infty}, m_{1}<m_{2}<\ldots$, are those functions of the system $\left\{\eta_{n}\right\}_{n=1}^{\infty}$ which do not belong to $\Phi_{1}$. We define a rearrangement of the system $\left\{\eta_{n}\right\}_{n=1}^{\infty}$ in the following manner: 
Let $\Pi=\left\{p_{k}\right\}_{k=1}^{\infty}$ be an increasing sequence of natural numbers satisfying the recurrent inequalities

$$
p_{s+1}>(s+1) m p_{s}, \quad p_{1}=1,
$$

for all $s=1,2, \ldots$ Then we put $\psi_{1}=\eta_{n_{1}}$ and

$$
\psi_{p_{k}}=\eta_{n_{k}} \quad \text { for all } k \geq 2
$$

and for any natural $j$ such that $p_{k-1}<j<p_{k}, p_{0}=1$, we put

$$
\psi_{j}=\eta_{m_{j-k}} \text {. }
$$

Denote the resulting system by $\Psi$.

Further we have to apply the following simple statement.

Lemma 2. Let $d \geq 1$ and $U \in \mathbf{A}(m, d)$. If $s \geq \max (2, d)$ and $R \geq p_{s}$, then $\left[0, p_{s-1}\right]^{m} \subset{ }^{R} U$.

Proof. Note that

$$
(\underbrace{0, \ldots, 0}_{j-1}, p_{s} / d, \underbrace{0, \ldots, 0}_{m-j}) \in{ }^{R} U \quad \text { for every } j \geq 1 .
$$

By (28),

$$
(\underbrace{0, \ldots, 0}_{j-1}, m p_{s-1}, \underbrace{0, \ldots, 0}_{m-j}) \in{ }^{R} U
$$

and by convexity $\left(p_{s-1}, \ldots, p_{s-1}\right) \in{ }^{R} U$, which implies the statement of Lemma 2.

Now let $d_{0} \geq 1$ be such that $A \in \mathbf{A}\left(m, d_{0}\right)$. Denote by $s_{0}$ the minimal natural number such that $s_{0} \geq \max \left(2, d_{0}\right)$. Take any $R \geq p_{s_{0}}$. Then we can find an $s=s(R)$ such that $p_{s} \leq R<p_{s+1}$. Let $\theta=\left\{j_{1}, \ldots, j_{q}\right\} \subset Z_{m}$ be a set for which

$$
U(R)=\left\{\mathbf{n}_{\bar{\theta}} \in\left[1, p_{s+1}-1\right]^{m-q}: \mathbf{n} \in{ }^{R} A \text { and } n_{k}=p_{s} \text { for } k \in \theta\right\}
$$

is not empty and put $\bar{\theta}=Z_{m} \backslash \theta=\left\{i_{1}, \ldots, i_{p}\right\} \neq \emptyset$.

Let

$$
B_{R}\left(f, \mathbf{x}_{\bar{\theta}}\right)=\sum_{\mathbf{n}_{\bar{\theta}} \in U(R)} b_{\mathbf{n}_{\bar{\theta}}} \psi_{\mathbf{n}_{\bar{\theta}}}\left(\mathbf{x}_{\bar{\theta}}\right)
$$

where

$$
b_{\mathbf{n}_{\bar{\theta}}}=\int_{[0,1]^{m}} f(\mathbf{t}) \prod_{\nu \in \theta} \psi_{p_{s}}\left(t_{\nu}\right) \psi_{\mathbf{n}_{\bar{\theta}}}\left(\mathbf{t}_{\bar{\theta}}\right) d \mathbf{t}, \quad \psi_{\mathbf{n}_{\bar{\theta}}}\left(\mathbf{x}_{\bar{\theta}}\right)=\prod_{\nu \in \bar{\theta}} \psi_{n_{\nu}}\left(x_{\nu}\right) .
$$

LEMMA 3.

$$
\sup _{R \geq p_{s_{0}}}\left|B_{R}\left(f, \mathbf{x}_{\bar{\theta}}\right) \cdot \operatorname{sign}\left[\psi_{\mathbf{n}_{\theta}}\left(\mathbf{x}_{\theta}\right)\right]\right|<\infty
$$

almost everywhere on $[0,1 / 2)^{m}$. 
Proof. According to (11) it suffices to prove the assertion on the sets $A_{r}^{m}$ for any $r \in \mathbb{N}$. By (12) we can find $s_{r} \geq s_{0}$ such that any function $\eta_{n_{k}}$ for $k \geq s_{r}$ vanishes on $[0,1 / 2) \backslash A_{r}$ and the functions $\eta_{m_{k}}$ vanish on $A_{r}$. Denote by $p^{\prime}>p_{s_{0}}$ the corresponding number for which any function $\psi_{p_{s}}$ for $p_{s} \geq p^{\prime}$ vanishes on $[0,1 / 2) \backslash A_{r}$ (cf. (29)) and the functions $\psi_{l}, l \geq p^{\prime}$, $l \notin\left\{p_{s}\right\}$, vanish on $A_{r}$. Evidently we obtain the assertion of Lemma 3 if we prove that

$$
\sup _{R \geq p^{\prime}}\left|B_{R}\left(f, \mathbf{x}_{\bar{\theta}}\right) \cdot \operatorname{sign}\left[\psi_{\mathbf{n}_{\theta}}\left(\mathbf{x}_{\theta}\right)\right]\right|<\infty
$$

almost everywhere on $A_{r}^{m}$.

We prove the above inequality by induction on the cardinality of $\bar{\theta}$. If $\operatorname{card} \bar{\theta}=1$ then the inequality follows easily from (22)-(24) and (27). Now suppose that (33) is true for any $\theta=\left\{j_{1}, \ldots, j_{q}\right\} \subset Z_{m}$ such that $\operatorname{card} \bar{\theta}<$ $\gamma<m$ and let us prove it for any $\theta=\left\{j_{1}, \ldots, j_{q}\right\} \subset Z_{m}$ such that $\operatorname{card} \bar{\theta}=\gamma$. Let

$$
U^{*}(R)=\left\{\mathbf{x}_{\bar{\theta}} \in[0,1]^{\gamma}: \mathbf{x} \in A \text { and } x_{k}=p_{s} / R \text { for } k \in \theta\right\} .
$$

According to Proposition $1, U^{*}(R) \in \mathbf{A}(\gamma)$. If $\alpha\left(U^{*}(R)\right) \cdot R \leq p^{\prime}$ then we can write the following estimate:

$$
\left|B_{R}\left(f, \mathbf{x}_{\bar{\theta}}\right) \cdot \operatorname{sign}\left[\psi_{\mathbf{n}_{\theta}}\left(\mathbf{x}_{\theta}\right)\right]\right|<\left(p^{\prime} M^{2}\right)^{\gamma}\|f\|_{1} .
$$

Otherwise, if $\alpha\left(U^{*}(R)\right) \cdot R>p^{\prime}$ we find $\sigma$ such that $p_{\sigma} \leq \alpha\left(U^{*}(R)\right) \cdot R<p_{\sigma+1}$ and let $\Gamma$ be the set of all those $\theta^{*} \subset \bar{\theta}$ for which

$$
V\left(\theta^{*}\right)=\left\{\mathbf{n}_{\overline{\theta^{*}}} \in\left[1, p_{\sigma+1}-1\right]^{\gamma-q^{*}}: R^{-1} \mathbf{n} \in U^{*}(R) \text { and } n_{k}=p_{\sigma} \text { for } k \in \theta^{*}\right\}
$$

is not empty, where $q^{*}=\operatorname{card} \theta^{*}$. Let $\sigma^{*} \leq \sigma-1$ be the maximal number such that $V\left(\theta^{*}\right) \supseteq\left[1, p_{\sigma^{*}}\right]^{\gamma-q^{*}}$ and let $\Omega_{\theta^{*}} \subset V\left(\theta^{*}\right)$ be the subset of all those vectors from $V\left(\theta^{*}\right)$ whose $k$ th coordinates, where $k \in \theta^{*}$, are equal to $p_{\sigma^{*}}$.

Thus we can write

$$
\begin{aligned}
\left|B_{R}\left(f, \mathbf{x}_{\bar{\theta}}\right)\right|= & \left|\sum_{\mathbf{n}_{\bar{\theta}} \in U(R)} b_{\mathbf{n}_{\bar{\theta}}} \psi_{\mathbf{n}_{\bar{\theta}}}\left(\mathbf{x}_{\bar{\theta}}\right)\right| \leq\left|\sum_{\left\|\mathbf{n}_{\bar{\theta}}\right\|_{\infty} \leq p_{\sigma-1}} b_{\mathbf{n}_{\bar{\theta}}} \psi_{\mathbf{n}_{\bar{\theta}}}\left(\mathbf{x}_{\bar{\theta}}\right)\right| \\
& +\sum_{\theta^{*} \in \Gamma}\left|\sum_{\mathbf{n}_{\bar{\theta}} \in \Omega_{\theta^{*}}} b_{\mathbf{n}_{\bar{\theta}}} \psi_{\mathbf{n}_{\bar{\theta}}}\left(\mathbf{x}_{\bar{\theta}}\right)\right| .
\end{aligned}
$$

It is evident that card $\Gamma \leq C_{m}$, where $C_{m}>0$ depends only on $m$. By the induction hypothesis we have

$$
\sup _{R \geq p^{\prime}}\left|\sum_{\mathbf{n}_{\bar{\theta}} \in \Omega_{\theta^{*}}} b_{\mathbf{n}_{\bar{\theta}}} \psi_{\mathbf{n}_{\bar{\theta}}}\left(\mathbf{x}_{\bar{\theta}}\right)\right|<\infty .
$$

Hence by (27), the proof of Lemma 3 is finished. 
To finish the proof of Theorem 4 we write

$$
\begin{aligned}
S_{A, R}^{\Psi}(f)(\mathbf{x}) & =\sum_{\mathbf{n} \in R_{A}} a_{\mathbf{n}}(f) \psi_{\mathbf{n}}(\mathbf{x}) \\
& =\sum_{\|\mathbf{n}\| \leq p_{s-1}} a_{\mathbf{n}}(f) \psi_{\mathbf{n}}(\mathbf{x})+\sum_{\|\mathbf{n}\|>p_{s-1}, \mathbf{n} \in R^{R} A} a_{\mathbf{n}}(f) \psi_{\mathbf{n}}(\mathbf{x}),
\end{aligned}
$$

where $p_{s} \leq R<p_{s+1}$. If $\Gamma^{*}$ is the set of all $\theta=\left\{j_{1}, \ldots, j_{q}\right\} \subset Z_{m}$ for which (31) holds then the second sum on the right side of the above equality can be estimated by

$$
\sum_{\theta \in \Gamma^{*}}\left|\sum_{\mathbf{n} \in \bar{\Omega}_{\theta}} a_{\mathbf{n}}(f) \psi_{\mathbf{n}}(\mathbf{x})\right|
$$

where $\bar{\Omega}_{\theta} \subset{ }^{R} A$ is the subset of all those vectors from ${ }^{R} A$ whose $m_{k^{-}}$ coordinates, where $k \in \theta$, are equal to $p_{s}$. By (15) we have

$$
\lim _{s \rightarrow \infty}\left\|\psi_{p_{s}}\right\|_{L^{\infty}[0,1 / 2]}=0 .
$$

Hence, by Lemma 3 we see that almost everywhere on $[0,1 / 2]^{m}$,

$$
\limsup _{R \rightarrow \infty} \sum_{\mathbf{n} \in \bar{\Omega}_{\theta}} a_{\mathbf{n}}(f) \psi_{\mathbf{n}}(\mathbf{x})=0 .
$$

But card $\Gamma^{*} \leq C_{m}$, where $C_{m}>0$ depends only on $m$. Thus we deduce the convergence almost everywhere on $[0,1 / 2]^{m}$ of the partial sums

$$
S_{A, R}^{\Psi}(f)(\mathbf{x})
$$

from the convergence almost everywhere on $[0,1 / 2]^{m}$ of the first sum on the right side of (34).

\section{References}

[1] M. I. D'yachenko and K. S. Kazaryan, Sets of convergence and divergence of multiple orthogonal series, Mat. Sb. 193 (2002), no. 9, 41-62 (in Russian).

[2] S. Kaczmarz and H. Steinhaus, Theorie der Orthogonalreihen, Warszawa, 1935; reprint, Chelsea, New York, 1951.

[3] K. S. Kazarian, Divergent orthogonal Fourier series, Mat. Sb. 182 (1991), 985-1008 (in Russian).

[4] - On some questions in the theory of orthogonal series, ibid. 119 (1982), 278-294 (in Russian).

[5] A. N. Kolmogoroff, Une série de Fourier-Lebesgue divergente presque partout, Fund. Math. 4 (1923), 324-328. 
[6] P. L. Ul'yanov, A. N. Kolmogorov and divergent Fourier series, Uspekhi Mat. Nauk 38 (1983), no. 4, 51-90 (in Russian).

Departamento de Matemáticas, C-XV

Universidad Autónoma de Madrid

28049 Madrid, Spain

E-mail: kazaros.kazarian@uam.es

Institute of Mathematics

National Academy of Sciences

Marshal Bagramyan Av. 24-B

375019 Yerevan, Armenia
Department of Function Theory Faculty of Mechanics and Mathematics Moscow State University Vorobevy Gory 119899 Moscow, Russia 